\title{
Chapter 10 \\ Summary of the Abstract Setting to get the Equations of Motion
}

\begin{abstract}
The abstract setting to get the equations of motion from the principle of virtual work is describe. The linear space of the virtual velocities and the deformation operator are the important elements. They are not chosen once for all. Engineers are free to choose them depending on their problems. Examples of such choices are given in Part II.
\end{abstract}

The basic elements are the linear space of the velocities $\mathcal{V}$ and the linear mapping, the linear deformation operator $\mathfrak{D}$, from $\mathcal{V}$ into an other linear space $\mathcal{D}$, the linear space of the velocities of deformation.

The external forces are abstract concepts, they are elements of the dual space $\mathcal{V}^{*}$. Their work is defined for any $V \in \tilde{\mathcal{V}}$ where $\tilde{\mathcal{V}}$ is the space of the special bounded variation functions with respect to time with value in $\mathcal{V}$.

The internal forces are also abstract concepts: they are elements of the dual space $\mathcal{D}^{*}$. Their work is defined for any $\mathfrak{D}(V)$ with $V \in \tilde{\mathcal{V}}$. They have neither power nor work with the rigid system velocities of the subspace $\mathcal{R} \subset \mathcal{V}$, i.e., with $\mathfrak{D}(\mathcal{R})=0$.

Both linear space $\mathcal{V}$ and deformation operator $\mathfrak{D}$ are chosen by the engineer. They are not imposed by any mechanical axiom. The deformation operator is not chosen once for all. It results that there is not only one type of internal forces.

In the sequel, we give examples involving:

- the description of the system;

- the choice of the velocities;

- the choice of the velocities of deformation, i.e., the choice of the deformation operator $\mathfrak{D}$.

With these choices the prediction of the motion is possible provided the constitutive laws relating the internal forces and the deformations are chosen. We do not investigate the choice of constitutive laws but we give some examples to illustrate the presentation. 